\title{
Hadron Production measurements at the NA61/SHINE experiment for the T2K Neutrino Flux Prediction
}

\section{Davide Sgalaberna* for the NA61/SHINE Collaboration}

ETH Zurich, Switzerland

E-mail: davide.sgalaberna@cern.ch

The largest source of uncertainty on the initial neutrino flux in modern accelerator neutrino experiments is the poor knowledge on the production of hadrons that decay into neutrinos. T2K is a long baseline neutrino experiment that aims to precisely measure the parameters of the PMNS matrix via the $v_{\mu} \rightarrow v_{e}$ appearance and $v_{\mu}$ disappearance as well as to look for the first indication of $\mathrm{CP}$ violation in the leptonic sector. The required total systematic uncertainty on the neutrino flux as low as $5 \%$ can hopefully be achieved with high precision hadron production measurements, performed by the dedicated auxiliary NA61/SHINE experiment at the CERN SPS. Production of hadrons in $31 \mathrm{GeV} / \mathrm{c}$ proton interactions on carbon is measured with a thin target (4\% of the nuclear interaction length) to study the primary interactions and with a T2K replica target (1.9 interaction length) to investigate re-interactions in the long target. The low statistic pilot data-set taken in 2007 was used to measure hadron multiplicities with the thin target and to demonstrate the capabilities of the spectrometer with the T2K replica target. High statistics 2009 and 2010 runs have been used to perform precise measurements. The latest 2009 results on charged pion, kaon and proton spectra are presented and experimental data are compared to model predictions. The re-weighting procedure used to tune the T2K neutrino flux is presented as well. This method will be very important also for the future neutrino long-baseline experiments for which a precision of about $2 \%$ on the flux knowledge is required for the discovery of $\mathrm{CP}$ violation in the lepton sector.

16th International Workshop on Neutrino Factories and Future Neutrino Beam Facilities - NUFACT2014, 25 -30 August, 2014

University of Glasgow, United Kingdom

\footnotetext{
* Speaker.
} 


\section{The NA61/SHINE experiment}

One of the main problems for the modern accelerator neutrino experiments is the large source of uncertainty on the initial neutrino flux due to the poor knowledge on the production of hadrons that decay into neutrinos. T2K (Tokai to Kamioka) [2] is a long baseline neutrino experiment which aims to precisely measure the parameters of the PMNS matrix via the $v_{\mu} \rightarrow v_{e}$ appearance and $v_{\mu}$ disappearance and to search for $\mathrm{CP}$ violation in the lepton sector [3, 4], requiring a total systematic uncertainty on the neutrino flux of about 5\%. This can be achieved by performing precision hadron production measurements at NA61/SHINE (SPS Heavy Ion and Neutrino Experiment), a fixed target experiment at the CERN SPS, which consists of a large acceptance detector with very good capabilities of momentum, charge and mass measurements [1]. A proton beam at the T2K energy, $31 \mathrm{GeV} / \mathrm{c}$, is used. The primary interactions are measured using a thin carbon target $(2 \mathrm{~cm}$ long, $4 \%$ of the nuclear interaction length), while a T2K replica target $(90 \mathrm{~cm}$ long and 1.9 interaction length) is necessary to measure the re-interactions of the produced hadrons (secondary interactions).

A low statistic pilot data-set was taken in 2007 to measure the hadron multiplicities with the thin target and to test the method developed for the measurement with the long target [5, 6, 7]. High statistics 2009 run data-set have been used to perform precise measurements.

Almost all the T2K phase space is covered by the 2009 data set, which improved the coverage with respect to the 2007 data set, as shown in fig. 1. Furthermore new particle spectra, like protons, $K_{S}^{0}$ and $\Lambda$ have been measured.

\section{Derivation of the spectra}

The particle spectra are derived by measuring the number of particles $\alpha$ in momentum and azimuthal angle $(p-\theta)$ intervals with inserted $\left(\Delta n_{\alpha}^{I}\right)$ and removed $\left(\Delta n_{\alpha}^{R}\right)$ target, which allows to measure the out-of-target interactions background. The differential cross section is performed using

$$
\frac{d^{2} \sigma_{\alpha}}{d p d \theta}=\frac{\sigma_{t r i g}}{1-\varepsilon}\left(\frac{1}{N^{I}} \frac{\Delta n_{\alpha}^{I}}{\Delta p \Delta \theta}-\frac{\varepsilon}{N^{R}} \frac{\Delta n_{\alpha}^{R}}{\Delta p \Delta \theta}\right)
$$

which subtracts the background estimated using the data. $\alpha$ is the particle type (e.g. $\pi^{-}$). $N^{I}$ and $N^{R}$ are the numbers of events selected with target inserted and removed. $\Delta p(\Delta \theta)$ is the bin size in momentum (polar angle), and $\varepsilon=0.123 \pm 0.004$ is the measured ratio of the interaction probabilities with removed and inserted target. $\sigma_{\text {trig }}$ is the measured trigger cross section, related to the number of protons on target interactions, which is

$$
\sigma_{\text {trig }}=305.7 \pm 2.7 \text { (stat) } \pm 1.0 \text { (det) } \mathrm{mb}
$$

The particle spectra normalized to the mean particle multiplicity in production interactions is calculated as

$$
\frac{d^{2} n_{\alpha}}{d p d \theta}=\frac{1}{\sigma_{p r o d}} \cdot \frac{d^{2} \sigma_{\alpha}}{d p d \theta}
$$

where $\sigma_{\text {prod }}$ is the measured cross section for production processes which normalizes the spectra.

The production cross section has been measured with the 2009 run data set: 

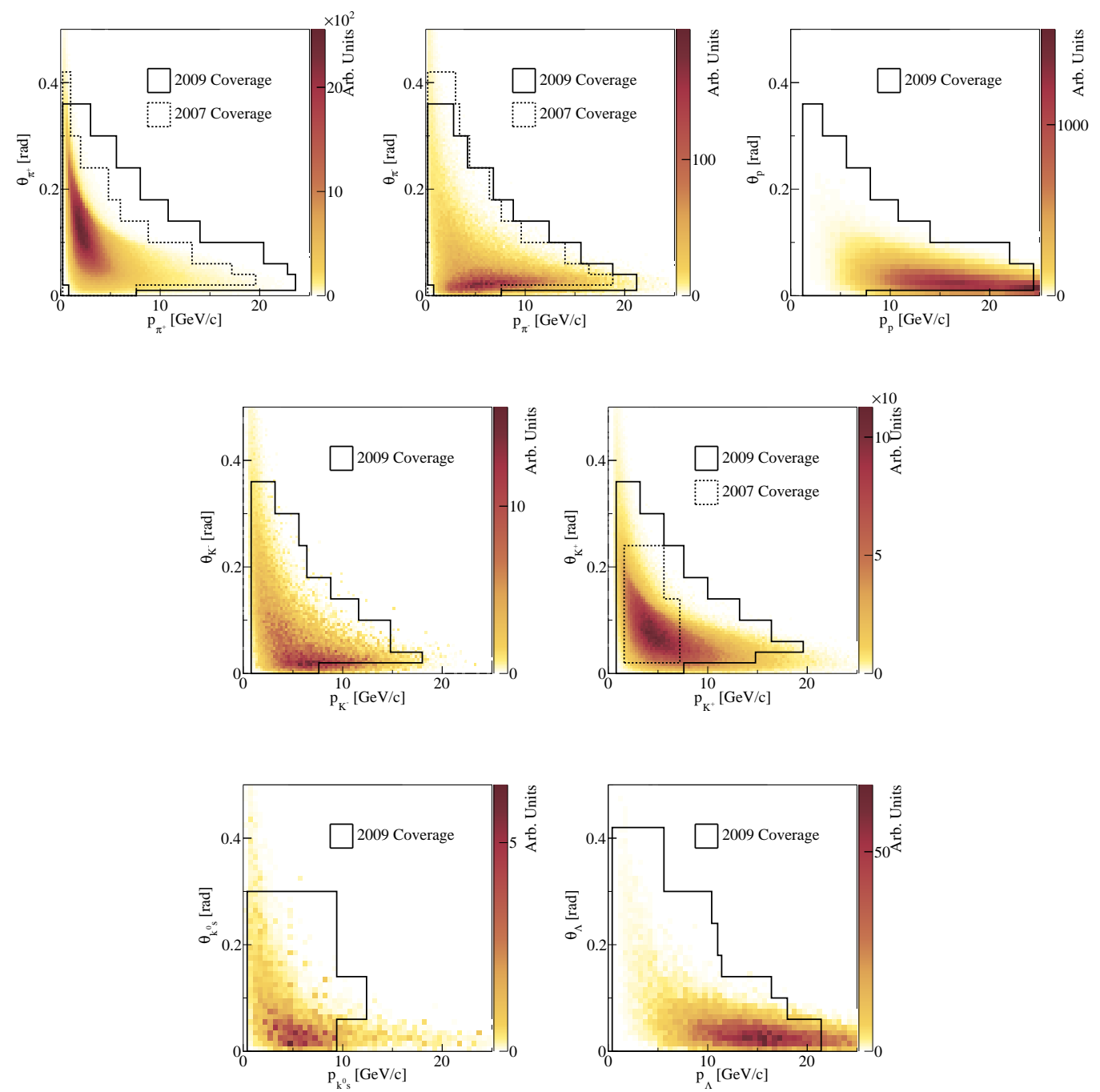

Figure 1: From left to right and up to bottom, the $p-\theta$ phase space of $\pi^{+}, \pi^{-}$, protons, $K^{+}, K_{S}^{0}$ and $\Lambda$ with positive focusing of the horn currents, contributing to the flux expected at the T2K far detector, are shown. The region covered by the NA61/SHINE measurement is overlaid for the 2009 (solid line) and 2007 (dashed line) measurement.

$$
\sigma_{\text {prod }}=230.8 \pm 2.8(\mathrm{stat}) \pm 2.5(\mathrm{det}){ }_{-2.8}^{+4.2}(\mathrm{mod}) \mathrm{mb}
$$

In fig. 2 the measured production cross section is compared with the other measurements available in literature.

\section{Charged hadrons spectra}

The multiplicities of the charged hadrons (pions, kaons and protons) are measured exploiting the energy loss informations given by the TPCs as well as using the particle time of flight measured by the ToF detector, in order to have a better particle identification above $1 \mathrm{GeV} / \mathrm{c}$. MC 


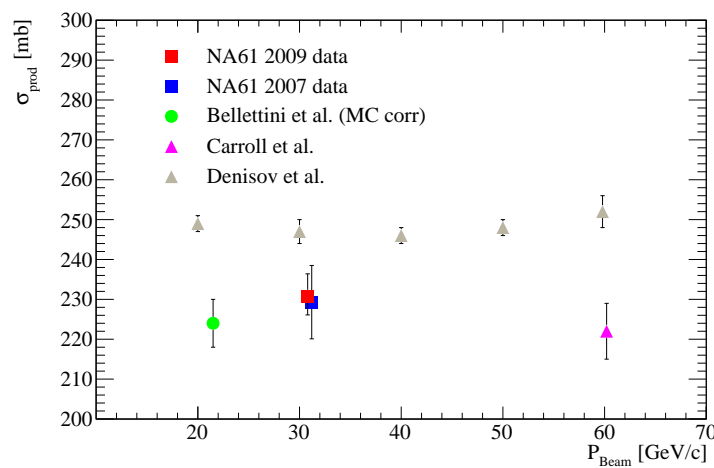

Figure 2: A comparison of the measured production cross section with previously published results at different momenta. Carroll et al. (pink empty circle) [8], Denisov et al. measurements (grey triangles) [9] and Bellettini et al. (green full circle) [10], that measured the inelastic cross section and the quasi-elastic component has been estimated with MC and subtracted. NA61/SHINE measurements with 2007 (blue full square) and 2009 (red full square) data samples are shown as well.
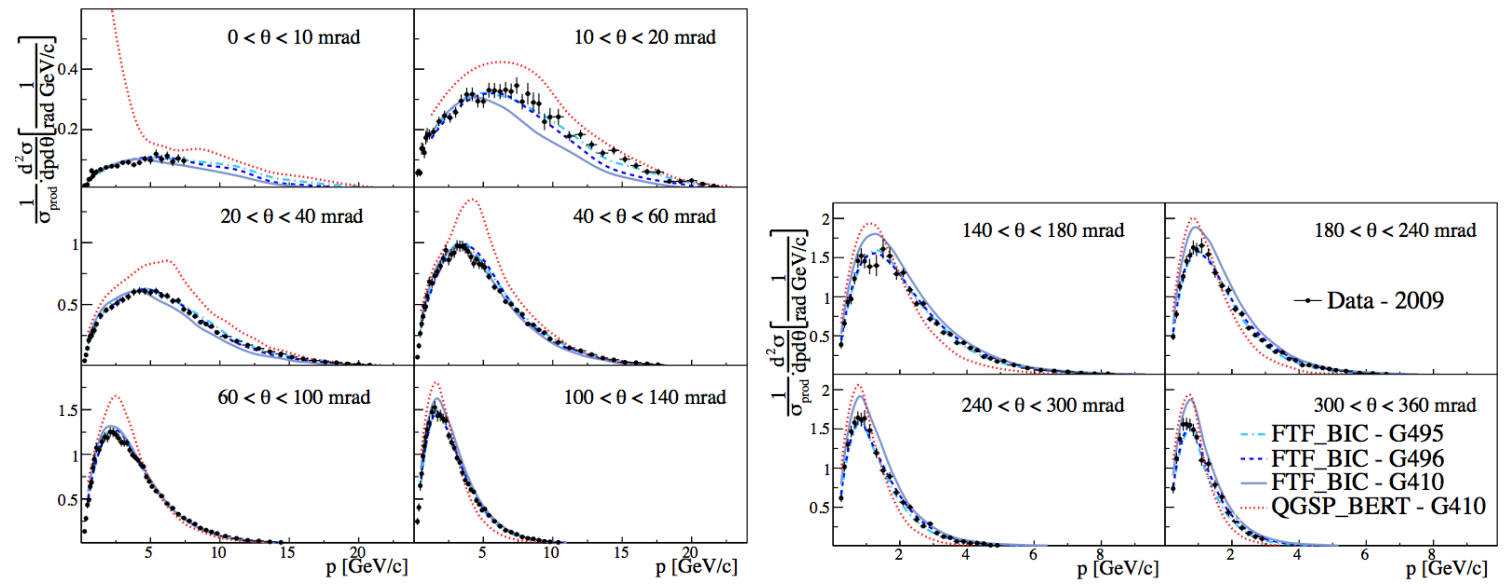

Figure 3: Distribution of $\pi^{+}$multiplicities produced in proton-carbon interactions at $31 \mathrm{GeV} / \mathrm{c}$ in different intervals of polar angle $\theta$. Error bars indicate the statistical uncertainty. Data (black points) are overlapped by the expected MC estimated using the physics list FTF_BIC for different versions of Geant4 (9.5, 9.6 and 10.0) as well as the QGSP_BERT physics list with the Geant4 10.0 version [12].

corrections which take into account the geometrical acceptance, the reconstruction efficiency, the contamination of other particles, secondary interactions and weak decays ("feed down correction"), are applied. In fig. 3 and fig. 4 the measured normalized spectra of positively charged pions and kaons are shown.

The statistical uncertainties have been reduced by a factor of three with respect to the results obtained with the 2007 run data. Also the systematic uncertainties are reduced by a factor of two. The largest contributions come from the uncertainty on the particle identification and the feed-down corrections, due to the decay of neutral strange particles $\left(V^{0}\right)$ such as $K_{S}^{0} \rightarrow \pi^{+} \pi^{-}$and $\Lambda \rightarrow p \pi^{-}$, which consist of the main source of systematic for $\pi^{+}, \pi^{-}$and protons. 

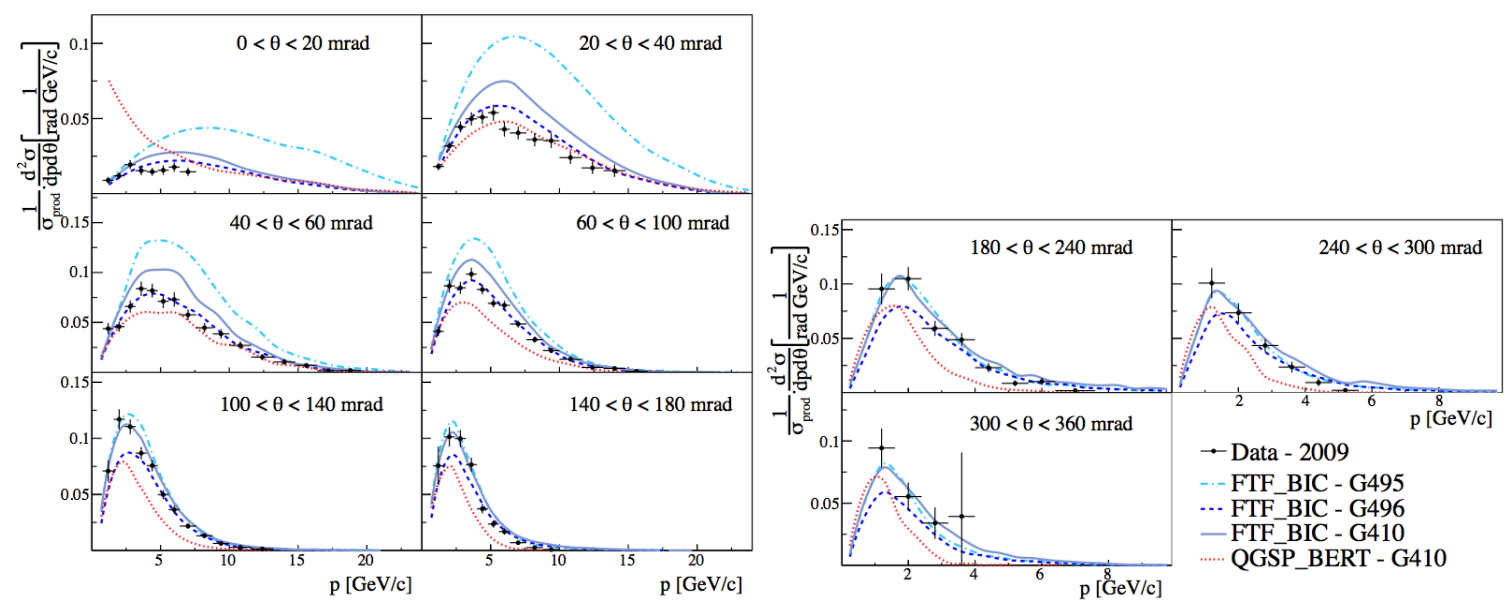

Figure 4: Distribution of $\mathrm{K}^{+}$multiplicities produced in proton-carbon interactions at $31 \mathrm{GeV} / \mathrm{c}$ in different intervals of polar angle $\theta$. Error bars indicate the statistical uncertainty. Data (black points) are overlapped by the expected MC estimated using the physics list FTF_BIC for different versions of Geant4 (9.5, 9.6 and 10.0) as well as the QGSP_BERT physics list with the Geant4 10.0 version [12].

\section{4. $V^{0}$ multiplicities}

In order to reduce the systematic uncertainty of the feed-down corrections, the spectra of the produced $K_{S}^{0}$ and $\Lambda$ have been measured. Furthermore most of the T2K $v_{e}$ flux at high energy is produced by $K_{L}^{0} \rightarrow \pi^{-} v_{e} e^{+}$and the measured $K_{S}^{0}$ spectra can be used to constrain this component. In fig. 5 the measured spectra of $K^{0}$ and $\Lambda$ normalized to the total production cross section are shown.

\section{Long target analysis}

The hadron spectra measured with the thin target data can constrain the expected T2K neutrino flux only up to about $60 \%$. Indeed the tertiary component, which consists of hadrons produced by interactions of secondary particles in the target or outside, produces about $40 \%$ of the neutrino flux. This component can be measured by analyzing the T2K replica target data.

Pions multiplicities, exiting the T2K replica target surface in five longitudinal bins of $18 \mathrm{~cm}$ each plus the downstream face, are measured. The method was successfully tested with the 2007 run data but the statistics was not enough to tune the T2K neutrino beam flux. The high statistics 2009 data set allows to perform a reliable measurements of the tertiary components.

The statistical uncertainty is between $5 \%$ and $8 \%$ and the systematic uncertainty is about $5 \%$ for particles exiting the central bins of the target while for the upstream and downstream faces it is about $14 \%$. The combination of the spectra measured with both the thin and long target allows to constrain the T2K neutrino flux up to the $90 \%$.

\section{Tuning of the T2K neutrino beam}

The hadron spectra measured at NA61/SHINE are used to tune the T2K expected neutrino flux, which is estimated with MC simulations: proton interactions in the target are simulated with 

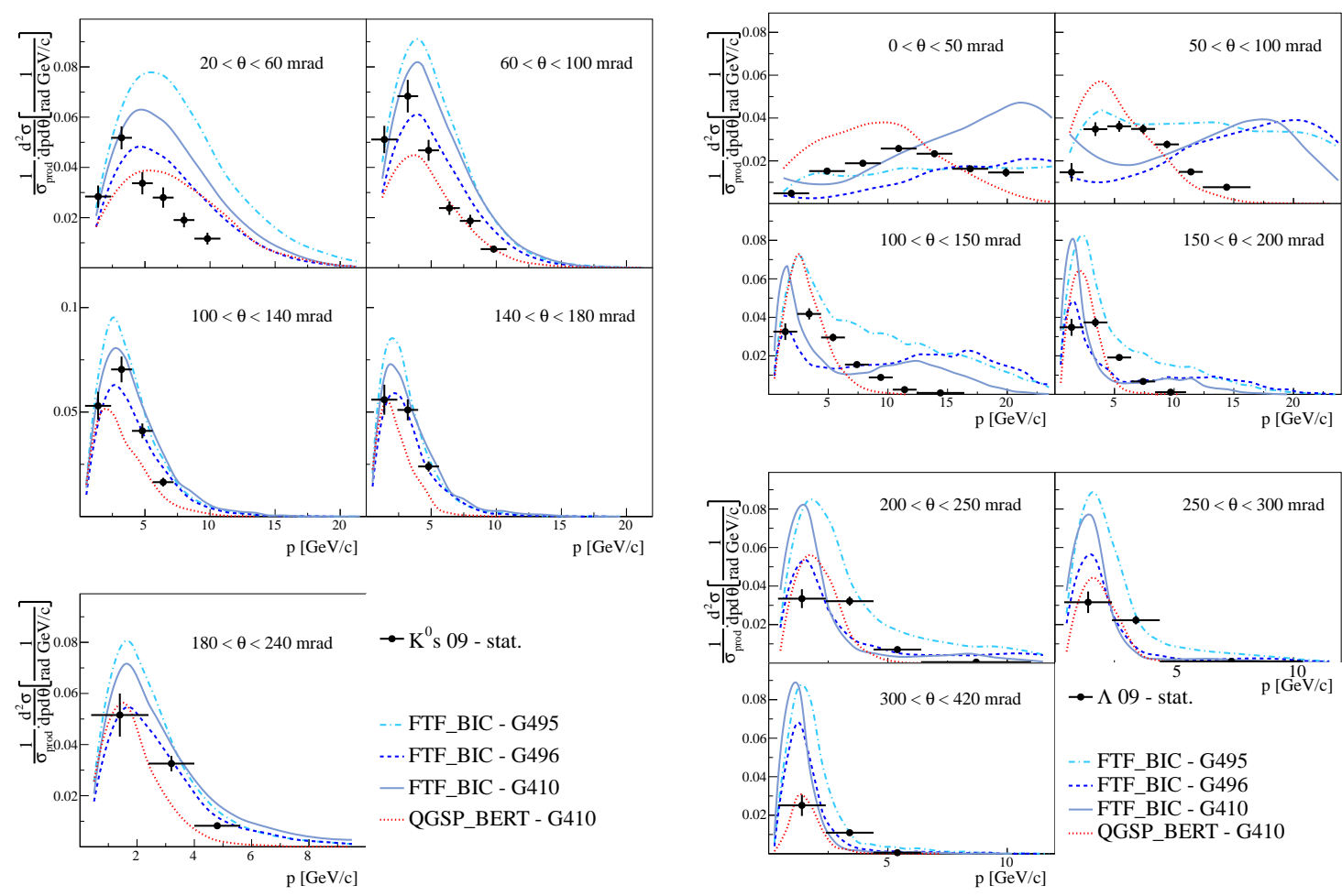

Figure 5: Distributions of $K_{S}^{0}$ (left) and $\Lambda$ (right) multiplicities produced in proton-carbon interactions at $31 \mathrm{GeV} / \mathrm{c}$ in different intervals of polar angle $\theta$ are shown. An invariant mass fit is performed on the raw yields of the tracks that fulfill the $V^{0}$ topology. Error bars indicate the statistical uncertainty. Data (black points) are overlapped by the expected MC estimated using the physics list FTF_BIC for different versions of Geant4 (9.5, 9.6 and 10.0) as well as the QGSP_BERT physics list with the Geant4 10.0 version [12].

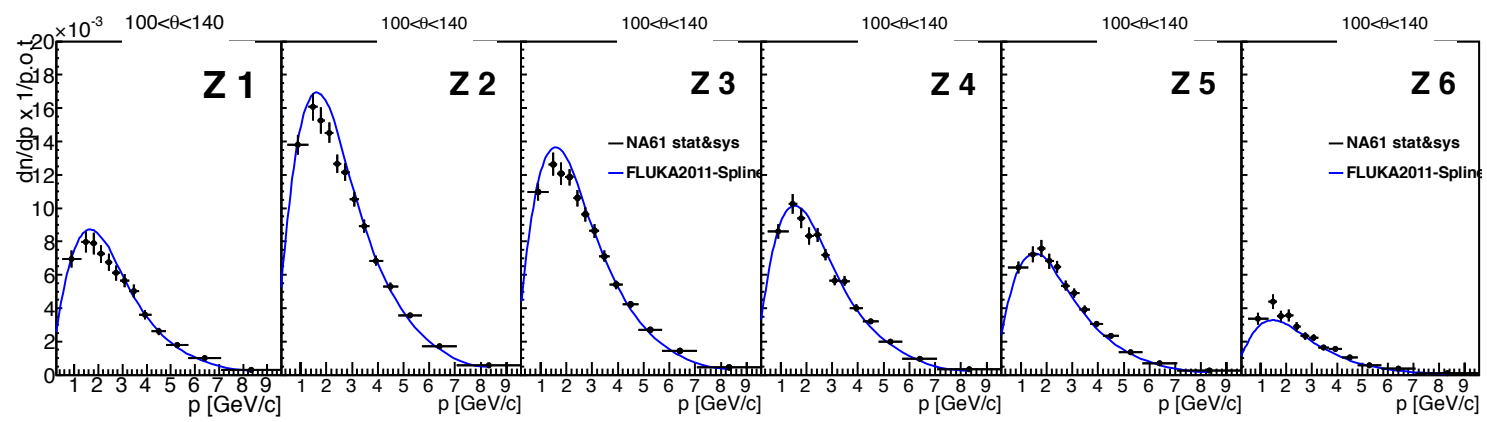

Figure 6: The spectra of $\pi^{+}$measured with the 2009 long target data in each target bin is shown as a function of the momentum for $100<\theta<140 \mathrm{mrad}$. Data (black dots) are overlapped to the FLUKA 2011 expectation $[13,14]$. Both statistical and systematic uncertainties are taken into account. 
the Fluka generator, the propagation in the beam-line is estimated using Geant3 interfaced with the GCALOR generator. For each kind of particle $i$ the weights are calculated as the ratio between the external data, with priority given to NA61/SHINE measurements, and T2K MC expectation in each $p-\theta$ bin:

$$
W\left(p_{i}, \theta_{i}, p_{0}, A\right)=\frac{\left[\frac{d n_{i}}{d p_{i}}\left(p_{i}, \theta_{i}, p_{0}, A\right)\right]_{\text {data }}}{\left[\frac{d n_{i}}{d p_{i}}\left(p_{i}, \theta_{i}, p_{0}, A\right)\right]_{M C}}
$$

where $p_{0}$ and $A$ are respectively the momentum of the interaction and the atomic number. The scaling hypothesis is used to extrapolate and re-weight the interactions that occur in different materials or in the regions of the phase space not covered by the external measurements. A detailed description of the procedure is given in [11].

The procedure has been successfully tested with the 2007 pilot run. After the flux tuning the total uncertainty was reduced from about $30 \%$ down to about $12 \%$, though still dominated by the hadron production. In the next future also the 2009 run data will be used to tune the T2K neutrino flux, further reducing the total flux systematic uncertainty.

\section{Conclusions}

The measurements of the hadron spectra at NA61/SHINE performed with the 2009 run data with the same proton beam and carbon target as at $\mathrm{T} 2 \mathrm{~K}$ are presented. They will be used to constrain the T2K neutrino flux for the next oscillation analyses. The phase space is better covered than with the 2007 run data and new particle spectra, such as protons, $K_{S}^{0}$ and $\Lambda$ are measured. Furthermore both statistical and systematic uncertainties have been reduced by a factor of 2-3.

New complementary analyses of the thin target 2009 data set which measure the charged pions spectra also for momenta below $1 \mathrm{GeV} / \mathrm{c}$ will be released soon.

The $90 \%$ of the T2K neutrino flux can be constrained by combining the measured spectra based on the thin target and long target data. The statistical uncertainties will be further reduced when also the 2010 run data set with the $\mathrm{T} 2 \mathrm{~K}$ replica target will be analyzed.

For the future neutrino long baseline experiments (LBNO-LAGUNA, LBNF, HyperKamiokande), which aim to measure $\mathrm{CP}$ violation in the leptonic sector, a total uncertainty on the neutrino flux prediction as low as $2 \%$ is mandatory. Hence it will be necessary to perform precise measurements of the hadron production.

\section{References}

[1] N. Abgrall et al, JINST 9 P06005 (2014).

[2] K. Abe et al., Nucl. Instrum. Meth. A 659, 106 (2011).

[3] K. Abe et al. Phys. Rev. Lett. 112, 181801 (2014)

[4] K. Abe et al. Phys. Rev. Lett. 112, 061802 (2014)

[5] N. Abgrall et al., Phys. Rev. C 84, 034604 (2011).

[6] N. Abgrall et al., Phys. Rev. C 85, 035210 (2012).

[7] N. Abgrall et al, Nucl. Instrum. Meth. A 701, 99 (2013). 
[8] A. S. Carroll et al., Phys. Lett. B 80, 319 (1979)

[9] S. P. Denisov et al., Nucl. Phys. B 61, 62 (1973)

[10] G. Bellettini et al., Nucl. Phys. B 79, 609 (1966)

[11] K. Abe et al., Phys. Rev. D 87, 012001 (2013)

[12] S. Agostinelli et al., Nucl. Instrum. Meth. A 506, 250 (2003).

[13] A. Ferrari, P.R. Sala, A. Fasso“, and J. Ranft, "FLUKA: a multi-particle transport code", CERN 2005-10 (2005), INFN/TC_05/11, SLAC-R-773

[14] T.T. Bohlen, F. Cerutti, M.P.W. Chin, A. Fasso`, A. Ferrari, P.G. Ortega, A. Mairani, P.R. Sala, G. Smirnov, and V. Vlachoudis, "The FLUKA Code: Developments and Challenges for High Energy and Medical Applications", Nuclear Data Sheets 120, 211-214 (2014) 